Escuela de Ciencias Sociales y Humanidades, UNED, C.R.

URL: http://investiga.uned.ac.cr/revistas/index.php/espiga/index

ISSN: 1409-4002 • e-ISSN: 2215-454X

doi: http://dx.doi.org/10.22458/re.v16i0.1926

\title{
La educación a distancia como factor de inclusión social: UNED, 40 años democratizando la educación en Costa Rica
}

\author{
Édgar Castro-Monge*
}

Recibido: 12 de junio, 2017 - Aceptado: 17 de octubre, 2017

\section{RESUMEN}

La educación a distancia se ha convertido en una modalidad de enseñanza que brinda grandes oportunidades a los estudiantes en el mundo que, por mucho tiempo, no han tenido acceso a la educación superior convencional o presencial. En Costa Rica no se sabía nada de esta forma de estudiar hasta que, en el año 1977, se da la apertura de una universidad con la modalidad completamente a distancia. En este artículo se da conocer, resumidamente, la labor que durante 40 años ha realizado la Universidad Estatal a Distancia (UNED), con una visión de inclusión social.

Palabras claves: Inclusión social, educación a distancia, cobertura, equidad, calidad.

\section{Introducción}

Es una realidad que las universidades en el mundo deben innovar continuamente, pensando en cómo adaptarse a los nuevos tiempos y a los cambios de las tecnologías de información y comunicación. El mundo ha cambiado, para bien en muchos casos, pero lamentablemente, la brecha entre la pobreza y la riqueza cada vez es mayor; día con día hay más pobres en el mundo; actualmente, existen menos oportunidades reales para las personas de bajos recursos.

\section{Formato de citación según APA}

Castro-Monge, E. (2017). La educación a distancia como factor de inclusión social: UNED, 40 años democratizando la educación en Costa Rica. Revista Espiga, 16(Número Especial), 8-39. doi: http://dx.doi.org/10.22458/re.v16i0.1926

Formato de citación según Chicago

Castro-Monge, Édgar. «La educación a distancia como factor de inclusión social: UNED, 40 años democratizando la educación en Costa Rica». Revista Espiga 16, n. ${ }^{\circ}$ Especial (2017): 8-39. doi: http://dx.doi.org/10.22458/re.v16i0.1926

* Édgar Castro-Monge, Vicerrector de Planificación, Universidad Estatal a Distancia, Costa Rica. Correo electrónico: edcastro@uned.ac.cr 
Las universidades deben tener, como eje central de su quehacer, la calidad, tanto académica como en la gestión universitaria. Esta es la principal razón por la que continuamente las universidades deben analizar su modelo educativo para adecuarse a las nuevas necesidades de las personas y volverse inclusivas para brindar oportunidades educativas a quienes más lo necesitan.

La educación a distancia ofrece oportunidades a todas las personas que, por diferentes razones, no han tenido acceso a la educación superior convencional. Las universidades con modalidad presencial en el mundo no pueden quedarse cruzadas de brazos; deben apostar por la educación a distancia y en línea, ya que brinda grandes ventajas para cumplir con el objetivo de inclusión social.

Por su parte, las tecnologías de la información y la comunicación (TIC) pueden contribuir al acceso universal en la educación. Las TIC, en el presente, son herramientas que deben aprovecharse para la enseñanza y aprendizaje de calidad.

Pero no todo es positivo cuando de TIC se habla. La brecha digital en los países también es cada vez mayor, por lo que se debe tener cuidado para que estas no se conviertan más bien en un factor de exclusión social. Por esta brecha digital, las universidades deben buscar los medios para hacer llegar esas tecnologías a los estudiantes que más lo necesitan.

También, las TIC son una fortaleza en el desarrollo profesional de los docentes. Por eso, los profesores deben adaptarse también a los nuevos tiempos; no pueden quedarse en el pasado, deben ser baluartes para la educación, pero con visión de inclusión social. El uso de estos recursos aumenta las múltiples posibilidades de interacción, entre profesores y alumnos y entre los mismos alumnos, ya que permiten desplegar mejores procesos y competencias pedagógico-didácticas.

Solo con nuevas ideas, con nuevas formas de realizar las labores diarias, el sistema educativo será más eficiente e inclusivo, por el bien de su razón de ser: el estudiante. Es aquí en donde la educación a distancia está jugando un papel preponderante en el mundo. En Costa Rica, la creación de la Universidad Estatal a Distancia (UNED) ha sido fundamental como factor de inclusión social.

\section{Creación de la UNED}

El 3 marzo de 1977 se dio la apertura de una de las instituciones públicas educativas que ha tenido más claro el tema de educación inclusiva. Una universidad comprometida con la misión de que la educación debía ser para todos, ofreciendo nuevas oportunidades de estudios en educación superior universitaria: la UNED. Primera universidad en Costa Rica y Centroamérica con una modalidad de estudio innovadora en el país, un modelo totalmente de educación a distancia.

Por aquellos años, los costarricenses no entendían cómo se podía estudiar a distancia. Las personas hablaban de una universidad por correspondencia, sin tener claro cómo se podía estudiar desde la casa. Para poder iniciar con su quehacer académico, se 
pusieron a la venta los sobres de matrícula, que contenían la documentación necesaria como requisito para poder matricularse en la nueva universidad.

Los pioneros de la UNED viajaban por todo el país buscando locales comerciales que hicieran el favor (así de claro: hacer el favor) de poner a la venta estos sobres de matrícula. Sin importar qué tipo de negocio era, lo importante era que estuvieran en todo el país y al alcance de todos los costarricenses, para que pudieran realizar la matrícula e iniciar sus estudios.

La UNED fue creada en la Administración del presidente Daniel Oduber Quirós, siendo Ministro de Educación don Fernando Volio Jiménez, bajo la Ley No. 6044 «Ley de Creación de la Universidad Estatal a Distancia (UNED)», publicada en el periódico oficial La Gaceta No.50 del 12 de marzo de 1977.

La UNED inicia su primer proceso de matrícula en 1978, en sus centros universitarios. En esa ocasión, tan solo 1200 estudiantes se matricularon; pero para eso, las autoridades universitarias tuvieron que tomar la decisión de ampliar el periodo de admisión, ya que, al cierre de la fase ordinaria, únicamente se habían matriculado poco más de 250 estudiantes.

Ante estos números, afloró la preocupación de los jerarcas, ya que las expectativas de matrícula eran más altas. Sin embargo, la situación más bien motivó a las autoridades universitarias a tomar las medidas necesarias para dar a conocer más a la UNED, con la intención de que la matricula siguiera creciendo en esta nueva institución.

Pero ¿Qué hacía diferente a esta universidad de las demás? ¿Cómo diferenciarse, cómo posicionarse en el país para tener credibilidad? Era una realidad que, cuando inició el quehacer académico de la UNED, no se creía en la educación a distancia ni tampoco se entendía cómo se podía estudiar bajo esta nueva modalidad.

Los creadores de la UNED lo tenían muy claro: no era posible que un país como Costa Rica, ejemplo de paz y democracia, siguiera excluyendo a personas por su pobreza o ubicación geográfica, debía existir una universidad que brindara oportunidades a lo largo de la vida, ya que solo con educación se podía dar acceso a las personas al trabajo y a las actividades sociales ¿Cómo podría entonces la nueva universidad luchar contra la exclusión educativa? Costa Rica no podía seguir marginando a muchas personas del acceso a la educación superior universitaria.

Bajo este contexto, la Universidad siguió creciendo en matrícula y abriendo nuevos centros universitarios en todo el país. También, siguió fortaleciendo su oferta académica con la apertura de más carreras, ampliando las oportunidades de educación y democratizando la educación superior en todo Costa Rica.

Fue con don Celedonio Ramírez como Rector, período 86-90, que el Consejo Universitario eliminó la venta de los sobres de matrícula y se decidió entregar gratuitamente al estudiante dicha documentación. Igualmente, se tomó la decisión de entregar los libros y demás materiales didácticos a los estudiantes junto con la matrícula. 
Desde el año de su creación, la UNED inició su oferta académica por semestres. Sin embargo, a partir del año 1995 fue aprobada la modalidad por cuatrimestre, por acuerdo del Consejo Universitario. Este entró en vigencia hasta el año 1996.

Así, transcurren 30 años del quehacer académico de la Universidad Estatal a Distancia y, mediante la Ley 8623 de la Asamblea Legislativa, fue declarada institución benemérita de la Educación y la Cultura de Costa Rica por sus grandes logros y aportes a la educación costarricense. Reconocimiento dado en la Presidencia de la República en San José, el primer día de noviembre de dos mil siete.

Por estas razones, cuarenta años después, se hace necesario recordar y dar un reconocimiento a los cinco rectores que durante cuatro décadas han sentado las bases de esta gran institución:

- Francisco Antonio Pacheco Fernández, desde 9 de abril 1977 hasta el 2 de setiembre de 1981.

- Chester Zelaya Goodman, desde 3 de setiembre de 1981 hasta el 27 de mayo de 1986.

- Celedonio Ramírez Ramírez, desde 28 de mayo de 1986 hasta el 9 de noviembre de 1999.

- Rodrigo Arias Camacho, desde el 10 de noviembre de 1999 hasta el 09 de noviembre de 2009.

- Luis Guillermo Carpio Malavasi, desde el 10 de noviembre de 2009 hasta el 09 de noviembre de 2019.

\section{Inclusión social y educativa como valores de la UNED}

La inclusión social es un proceso que buscar luchar contra la exclusión de todas las personas. La exclusión limita los derechos sociales para obtener un estándar básico de vida y se convierte en una barrera a la participación en las principales oportunidades sociales y ocupacionales de la sociedad ${ }^{1}$.

La exclusión social, entonces, se convierte en un proceso en el que las personas son marginadas y se les impide su completa participación en la sociedad en razón de su pobreza o falta de competencias básicas y oportunidades a lo largo de la vida; o como resultado de discriminación. La exclusión las aleja del trabajo, ingresos y oportunidades de educación, así como de redes y actividades sociales y comunales. Las personas excluidas tienen poco acceso a órganos de poder y toma de decisiones y se ven afectadas en su vida cotidiana por las decisiones que toman estos órganos ${ }^{2}$. Las dimensiones de la exclusión social se pueden clasificar en: educativa, laboral, económica, política, de género, diversidad cultural, entre otros.

1. Hilary Silver y SM Miller, «Social Exclusion. The European Approach to Social Disadvantage», Indicators, $\mathrm{n}^{\circ} 2$ (2003): 7-11.

2. Comisión Europea, 2003. 
Por su parte, la inclusión educativa, en su base principal, tiene como objetivo promover cambios educativos sistemáticos, que puedan llevar los valores a la acción. Estos incluyen: equidad, solidaridad, igualdad de oportunidades, no discriminación, consideración, entre otros ${ }^{3}$.

Con respecto a este concepto de inclusión educativa, se puede mencionar que encuentra su sentido al considerarse como un valor y derecho social inalienable que tienen todas las personas y, al mismo nivel, como otros que tienen que ver con el derecho a la vida o la igualdad ${ }^{4}$. Es por ello que la educación inclusiva, desde su perspectiva, pone el énfasis en los modos de superar tanto los procesos y las situaciones de abierta exclusión, segregación y marginación educativa que, a lo largo del tiempo, han vivido muchas personas en el mundo ${ }^{5}$.

En un sentido mucho más amplio, la UNESCO en el 2008 conceptualizó la educación inclusiva como aquel principio rector necesario para apoyar y reforzar la educación en todas las personas para el aprendizaje a lo largo de toda la vida, el desarrollo sostenible y el acceso a las oportunidades de aprendizaje, haciendo énfasis en un factor fundamental de igualdad en todos los niveles de la sociedad.

Vinculado con lo anterior, no se puede negar el gran papel que las universidades públicas con modalidad presencial han jugado en el fortalecimiento de la sociedad costarricense, ya que han brindado oportunidades a muchas personas. Sin embargo, las universidades con modalidad presencial tienen factores de exclusión educativa, iniciando con el examen de admisión. Además, su área de cobertura no abarca todo el país, por lo que muchos jóvenes quedan excluidos y no pueden optar por la educación superior universitaria. De esta manera, la educación inclusiva, pone principal énfasis en todas las formas necesarias para poder superar tanto los procesos y las situaciones de abierta exclusión que tengan que ver con segregación y marginación educativa que, por mucho tiempo, han vivido gran cantidad de personas en el mundo ${ }^{6}$.

Tomando en cuenta todo lo señalado anteriormente, se puede apreciar cómo la UNED ha podido cumplir con la misión que la Ley de Creación, le dio a la Universidad y que pretende suplir las carencias que otras modalidades de estudio presentan: «Ofrecer educación superior a todos los sectores de la población, especialmente a aquellos que por razones económicas, sociales, geográficas, culturales, etarias, de discapacidad o de género, requieren oportunidades para una inserción real y equitativa en la sociedad» ${ }^{7}$.

3. Tony Booth y Mel Ainscow, Index for inclusión. Developing leaning and participation in schools, ( $3^{\mathrm{a}} \mathrm{ed}$. Manchester: CSIE, 2011.

4. Gerardo Echeita, «Inclusión y exclusión Educativa: Voz y Quebranto». Revista Iberoamericana sobre Calidad, Eficacia y Cambio en Educación, n² 2. (2013):9-18.

5. María Antonieta Casanova y Miguel Ángel Cabra de Luna, Educación y personas con discapacidad. Presente y Futuro (Madrid, España: Fundación ONCE, 2009).

6. Casanova y Cabra de Luna, Educación y personas..., 2009.

7. Ley 6044 de Creación de la Universidad Estatal Distancia (La Gaceta núm.50 del 12 de marzo de 1977). 


\section{La UNED como una universidad inclusiva}

En el contexto anterior, se señala cómo la UNED ha logrado ejecutar su quehacer académico con una visión de inclusión educativa. Sí se hace necesario señalar que, para este autor, la educación superior en el mundo para cumplir con una educación inclusiva debe enfocarse en:

1. Acceso

2. Cobertura

3. Calidad en la academia

4. Calidad en la gestión universitaria como apoyo a la academia

5. Pertinencia en la oferta académica

6. Permanencia del estudiante

7. Uso de las TIC

8. Equidad

9. Internacionalización

Al cumplir con estas variables sí podría hablarse de educación inclusiva; también, se posibilita crear oportunidades, democratizar la educación y obtener alta credibilidad en la sociedad. Para detallar los logros de la UNED como institución inclusiva se hace necesario, primero, mencionar sus áreas sustantivas. Por lo general, se habla de tres: Docencia, Investigación y Extensión; sin embargo, hay otras áreas que no se pueden dejar de lado: Gestión Universitaria, Producción de Materiales y Vida Estudiantil. Como se verá a continuación, todas estas áreas sustantivas han sido fortalecidas con el transcurrir de los años y han logrado posicionar a la UNED no solo a nivel nacional sino, también, a nivel internacional.

La gestión universitaria, es fundamental como apoyo a la academia. Las universidades deben apostar por la calidad en la gestión universitaria. En tanto se actúe con eficacia y una visión de mejora continua, más beneficiada será la academia, que al final es la responsable de brindar educación a la razón de ser de la universidad: los estudiantes.

En segundo lugar, la producción de materiales se ha tomado siempre como parte de la Docencia; sin embargo, se ha convertido en área sustantiva de la universidad. La labor de la Editorial UNED y de la Dirección de Producción de Materiales ha caracterizado e identificado a la institución desde que fue creada. También, la ha diferenciado de otras universidades de educación a distancia que no cuentan con un proceso de producción de materiales didácticos; o bien, no poseen uno tan fortalecido como el de la UNED. Tampoco se puede dejar de mencionar la gran producción de materiales de línea cultural ofrecidos por la Editorial UNED como aporte a la sociedad costarricense.

De igual manera, ha sido fundamental la creación de la Dirección de Producción de Materiales que, además de la producción de materiales impresos y en línea, se encarga también de la producción de audiovisual y multimedial para estudiantes y el país en 
general. Muchas de estas producciones audiovisuales han ganado premios a nivel nacional e internacional.

Por último, es una realidad que uno de los pendientes de la UNED, en sus 40 años, es no contar con una Vicerrectoría de Vida Estudiantil. En la actual estructura, la Dirección de Asuntos Estudiantiles es parte de la mal llamada Vicerrectoría Ejecutiva (debería llamarse Vicerrectoría de Administración), a todas luces un error en la estructura de la Universidad en el tema de vida estudiantil. Vale mencionar que el Señor Rector Luis Guillermo Carpio ha puesto todo su empeño para que esto sea una realidad y se pueda concretar la nueva vicerrectoría lo más pronto posible.

Tomando en cuenta las nueve variables anteriores y sus áreas sustantivas, se describe entonces como la UNED ha logrado convertirse en una universidad inclusiva que ha brindado tantas oportunidades de educación superior a personas que jamás pensaron haber tenido esas posibilidades.

\section{Acceso a la educación}

El acceso a la educación contribuye a tener una sociedad consolidada social, política y económicamente y, en este sentido, las universidades públicas han jugado un papel trascendental en Costa Rica. De esta manera, todo el quehacer de esta nueva universidad debía girar alrededor de la misión establecida en su creación. Por eso, la primera gran estrategia de inclusión social, relacionada con el acceso, fue tomar la decisión de no tener examen de admisión. Así, para dar oportunidades a cualquier costarricense que tuviera el bachillerato de educación secundaria, el sobre de matrícula que se vendía a un precio muy cómodo, era la única documentación necesaria para que el estudiante realizara la matrícula.

Esa gran visión de inclusión se fortaleció con la creación, en 1978, de la antes aludida Editorial UNED. Su fin era producir los materiales didácticos que se les entregarían a los estudiantes para que pudieran ir a sus hogares, en todo el país, a estudiar. Este fue el inicio de lo que hoy es la editorial más prestigiosa de Centroamérica. Hoy en día no solo ha apostado por los libros didácticos; también, ha ofrecido a Costa Rica un acervo de línea cultural. Es necesario mencionar que el primer libro fue ¿Democracia en Costa Rica? Cinco opiniones polémicas, de los autores Chester Zelaya Goodman, Oscar Aguilar Bulgarelli, Daniel Camacho, Rodolfo Cerdas y Jacobo Shifter Sikolara.

\section{El Programa de becas como factor que influye en el acceso a la UNED}

Aun cuando la UNED es la universidad que menos recursos recibe del Estado Costarricense, ha realizado un gran esfuerzo para llevar a cabo un programa de becas como factor fundamental para brindar acceso a la educación superior a aquellas personas de muy bajos recursos. En la tabla número 1 se da a conocer la distribución del Fondo Especial para la Educación Superior Universitaria (FEES), en donde se nota que a la UNED le corresponde tan solo el 7.5 del FEES, a pesar de ser la segunda universidad del país con más población estudiantil, ya que atiende un promedio entre 31000 y 32000 estudiantes 
al año. A ese 7,5\% se le debe sumar 1,5\% como aporte del Fondo de Fortalecimiento de la Educación a Distancia, lo que lo convierte en un $9 \%$.

TABLA 1

Distribución del FEES Institucional Costa Rica

Universidad de Costa Rica (UCR)

Universidad Nacional (UNA)

Tecnológico de Costa Rica (TEC)

Universidad Estatal a Distancia (UNED)

Total

Universidad Técnica Nacional (UTN)

$$
\begin{gathered}
57,79 \% \\
23,40 \% \\
11,30 \% \\
7,51 \% \\
100 \%
\end{gathered}
$$

Sus recursos son incorporados en el Presupuesto Nacional.

Fuente: elaboración propia con datos de CONARE 2017.

Para ampliar el aspecto del problema de recursos económicos, debe señalarse que en la UNED por muchos años los recursos del FEES Institucional no alcanzaban para pagar la masa salarial, lo que ha obligado a la institución a realizar un esfuerzo institucional para sanear las finanzas, logrando que esa brecha se haya disminuido. Como ejemplo, al año 2012, la brecha negativa era de 4.044, 93 millones de colones pero, en el 2016, se logra disminuir a 1.225,43 millones de colones. Esto porque la masa salarial de la UNED al 2016 era de 38.757,49 millones de colones y el FEES institucional de 37.532,06 .

Aun cuando queda demostrado, con los datos anteriores, que los recursos recibidos por la UNED son limitados, el Programa de Becas, coordinado desde la Dirección de Asuntos Estudiantiles, ha realizado un gran aporte a los costarricenses que más lo necesitan. Esos datos se muestran en las siguientes tablas:

TABLA 2

Tipos de becas otorgadas por la UNED

\begin{tabular}{cl} 
Tipo de beca & \multicolumn{1}{c}{ Beneficio } \\
Beca A & $\begin{array}{l}100 \% \text { exoneración en el pago de las materias y de matrícula. Se otorga un apoyo } \\
\text { económico para gastos asociados de matrícula por cuatrimestre. }\end{array}$ \\
Beca B & $\begin{array}{l}100 \% \text { exoneración en el pago de las materias. } \\
\text { Beca C }\end{array}$ \\
$\begin{array}{cl}75 \% \text { exoneración en el pago de las materias. } \\
\text { Beca D }\end{array}$ & $50 \%$ exoneración en el pago de las materias. \\
Beca E & $25 \%$ exoneración en el pago de las materias. \\
\hline
\end{tabular}

Fuente: elaboración propia con datos DAES, 2017.

8. Luis Guillermo, Carpio Malavasi. «Informe de Labores UNED 2016» (Costa Rica: Universidad Estatal a Distancia), 2017. 
TABLA 3

Total de becas asignadas en el 2016

\begin{tabular}{cc} 
Periodo académico & Cantidad de becas \\
$2016-1$ & 7880 \\
$2016-2$ & 7989 \\
$2016-3$ & 8268 \\
TOTAL & 24137 \\
\hline
\end{tabular}

Fuente: elaboración propia con datos DAES, 2017.

Los dineros que la universidad no percibe por concepto de becas, responden a la exoneración con que se beneficia a los estudiantes según el tipo de beca que se le otorga.

TABLA 4

Dinero no percibido por la UNED por concepto de becas en el año 2016

\begin{tabular}{ccc}
\hline Periodo académico & Monto en colones no percibido & Equivalente en dólares \\
\hline $2016-1$ & $\mathbb{C} 776.042 .500,00$ & $1.410 .986,35$ \\
$2016-2$ & $\mathbb{C} 774.184 .000,00$ & $1.407 .607,27$ \\
$2016-3$ & $\mathbb{C} 849.952 .900,00$ & $1.545 .368,91$ \\
Total & $\mathbb{C} \mathbf{2 . 4 0 0 . 1 7 9 . 4 0 0 , 0 0}$ & $\mathbf{4 . 3 6 3 . 9 6 2 , 5 3}$ \\
\hline
\end{tabular}

Fuente: elaboración propia con datos DAES, 2017 (tipo cambio: 550 colones por dólar).

El presupuesto invertido en becas A, dirigidas a estudiantes en situación de pobreza extrema, durante el año 2016 y en el que se le entrega un monto económico a los estudiantes fue de $\$ \mathbf{4 1 9 . 0 1 0 . 9 0 0 , 0 0}$ (\$761.838.00).

TABLA 5

Cantidad de estudiantes por beca por centro universitario durante 2016

\begin{tabular}{lclc}
$\begin{array}{c}\text { Centro } \\
\text { Universitario }\end{array}$ & $\begin{array}{c}\text { Cantidad } \\
\text { Estudiantes }\end{array}$ & \multicolumn{1}{c}{$\begin{array}{c}\text { Centro } \\
\text { universitario }\end{array}$} & $\begin{array}{c}\text { Cantidad } \\
\text { Estudiantes }\end{array}$ \\
San José & 1306 & La Cruz & 94 \\
Quepos & 76 & Upala & 212 \\
Cartago & 662 & Liberia & 295 \\
Alajuela & 392 & Turrialba & 193 \\
San Carlos & 477 & Buenos Aires & 605 \\
Palmares & 889 & Estudiantes en el extranjero & 274 \\
Nicoya & 124 & Santa Cruz & 5 \\
\hline
\end{tabular}


Tabla 5 (Continuación)

\begin{tabular}{lclc}
$\begin{array}{c}\text { Centro } \\
\text { Universitario }\end{array}$ & $\begin{array}{c}\text { Cantidad } \\
\text { Estudiantes }\end{array}$ & \multicolumn{1}{c}{$\begin{array}{c}\text { Centro } \\
\text { universitario }\end{array}$} & $\begin{array}{c}\text { Cantidad } \\
\text { Estudiantes }\end{array}$ \\
Cañas & 177 & La Reforma & 99 \\
Puntarenas & 210 & Heredia & 95 \\
Ciudad Neily & 271 & Atenas & 402 \\
Osa & 119 & Tilarán & 46 \\
Limón & 186 & Monteverde & 68 \\
San Isidro & 824 & Puerto Jiménez & 31 \\
Siquirres & 150 & Desamparados & 27 \\
Guápiles & 345 & Pavón & 452 \\
Orotina & 74 & Talamanca & 59 \\
Sarapiquí & 155 & Acosta & 146 \\
Puriscal & 327 & Privados de libertad & 127 \\
San Vito & 269 & Sede interuniversitaria & 199 \\
Jicaral & 75 & & 24 \\
& & Total de estudiantes con beca & 10561 \\
\hline
\end{tabular}

Fuente: elaboración propia con dato DAES 2017.

\section{Acceso a la educación a poblaciones específicas}

El Acceso a la educación en la UNED se ha fortalecido al focalizar esfuerzos hacia algunas poblaciones específicas con grandes necesidades educativas. Por ejemplo, personas con alguna discapacidad, personas privadas de libertad, poblaciones indígenas y educación secundaria.

\section{Personas con alguna discapacidad}

La atención a las personas con alguna discapacidad es parte de la misión que acompaña a la UNED desde que fue creada. Es así como desde la Dirección de Extensión de la UNED se han creado importantes programas educativos como: Programa de Braille, Programa Educación Especial y el Programa Lengua de Señas Costarricense (LESCO).

En este mismo sentido, en la Escuela de Ciencias de la Educación, se oferta la carrera en Educación especial. Dentro de sus objetivos, propone apoyar la inclusión plena y el ejercicio de los derechos humanos de todas aquellas personas con necesidades educativas especiales; así como atender las áreas de estudio vinculadas, a su vez, cumplir con lo que establece la Ley 7600 de Igualdad de las Oportunidades para las Personas con alguna discapacidad en Costa Rica. Por su parte, desde la UNED, las diferentes instancias académicas y administrativas han llevado a cabo un programa para atender estas personas con alguna discapacidad, importante, trabajo que se refleja en las tablas siguientes. 
Muestra de ello, es el Programa de Atención a Estudiantes con Necesidades Educativas Especiales, de la Dirección de Asuntos Estudiantiles.

TABLA 6

Estudiantes de la UNED con alguna discapacidad a marzo 2017

Clasificado por discapacidad

\begin{tabular}{lccc}
\multicolumn{1}{c}{$\begin{array}{c}\text { Estudiantes con alguna } \\
\text { discapacidad }\end{array}$} & $\begin{array}{c}\text { Matriculados } \\
\text { I cuatrimestre 2017 }\end{array}$ & $\begin{array}{c}\text { Estudiantes con } \\
\text { alguna discapacidad }\end{array}$ & $\begin{array}{c}\text { Matriculados } \\
\text { I cuatrimestre 2017 }\end{array}$ \\
Visual / baja visión / ceguera & 47 & Emocional & 25 \\
Auditiva & 13 & Salud & 4 \\
Física & 17 & Sistémica & 15 \\
Deficit atencional & 11 & Aprendizaje & 264 \\
Múltiple & 13 & Motora & 16 \\
Total estudiantes & & & 426 \\
\hline
\end{tabular}

Fuente: elaboración propia con datos de Dirección de Asuntos Estudiantiles (2017).

TABLA 7

De personas con alguna discapacidad por centros universitarios a marzo (2017)

\begin{tabular}{lclc}
\multicolumn{1}{c}{ Centros universitarios } & $\begin{array}{c}\text { Matriculados } \\
\text { a marzo } 2017\end{array}$ & \multicolumn{1}{c}{ Centros universitarios } & $\begin{array}{c}\text { Matriculados } \\
\text { a marzo 2017 }\end{array}$ \\
Alajuela & 14 & Palmares & 90 \\
Atenas & 3 & Pavón & 1 \\
Buenos aires & 4 & Privados de libertad & 1 \\
Cañas & 2 & Puntarenas & 3 \\
Cartago & 43 & Puriscal & 13 \\
Ciudad neily & 1 & Quepos & 2 \\
Desamparados & 12 & San carlos & 26 \\
Guápiles & 7 & San isidro & 18 \\
Heredia & 38 & San josé & 87 \\
Jicaral & 2 & San marcos & 9 \\
La cruz & 3 & San vito & 5 \\
Liberia & 2 & Santa cruz & 1 \\
Limón & 5 & Sarapiquí & 3 \\
Monte verde & 2 & Siquirres & 4 \\
Nicoya & 6 & Tilarán & 3 \\
Orotina & 2 & Turrialba & 8 \\
Osa & 1 & Upala & 6 \\
Total & & & 426 \\
\hline
\end{tabular}

Fuente: Dirección de Asuntos Estudiantiles (2017). 


\section{Poblaciones indígenas}

El trabajo que ha realizado la UNED con las poblaciones indígenas ha sido muy importante como factor de inclusión social. Esto se puede reflejar en las tablas siguientes.

TABLA 8

Estudiantes indígenas matriculados al primer cuatrimestre de 2017 por región

\begin{tabular}{lcc}
\multicolumn{1}{c}{ Región } & Cantidad & $\%$ \\
Región Huetar Caribe & 151 & 43.5 \\
Región Brunca & 130 & 37.5 \\
Región Central Este & 29 & 8.4 \\
Región Central Oeste & 15 & 4.3 \\
Región Chorotega & 10 & 2.9 \\
Región Huetar Norte & 7 & 2 \\
Región Pacífico Central & 3 & 0.9 \\
Extranjeros & 2 & 0.6 \\
Total estudiantes & 347 & 100
\end{tabular}

Fuente: elaboración propia con datos de AGPI-AMI 2017.

TABLA 9

Estudiantes indígenas matriculados al primer cuatrimestre de 2017 por género

\begin{tabular}{|c|c|c|}
\hline Género & Cantidad estudiantes & Porcentaje \\
\hline Femenino & 220 & 63.4 \\
\hline Masculino & 127 & 36.6 \\
\hline Total & 347 & 100 \\
\hline
\end{tabular}

Fuente: elaboración propia con datos: AGPI-AMI 2017. 
TABLA 10

Estudiantes indígenas becado por condición socioeconómica al primer cuatrimestre 2017, según centro universitario

\begin{tabular}{lclc}
$\begin{array}{c}\text { Centro } \\
\text { Universitario }\end{array}$ & $\begin{array}{c}\text { Estudiantes indigenas } \\
\text { Becados por } \\
\text { Condición socioeconómica }\end{array}$ & Centro universitario & $\begin{array}{c}\text { Estudiantes indigenas } \\
\text { becados por condición } \\
\text { socioeconómica }\end{array}$ \\
San José & 9 & Pérez Zeledón & 10 \\
Quepos & 1 & Guápiles & 1 \\
Cartago & 3 & Puriscal & 20 \\
San Carlos & 7 & San Vito & 11 \\
Nicoya & 3 & Upala & 1 \\
Puntarenas & 1 & Turrialba & 16 \\
Ciudad Neily & 20 & Buenos Aires & 74 \\
Osa & 11 & Talamanca & 99 \\
Limón & 23 & & \\
TOTAL & & & 310 \\
\hline
\end{tabular}

Fuente: elaboración propia con datos Oficina de Atención Socioeconómica, DAES, mayo 2017.

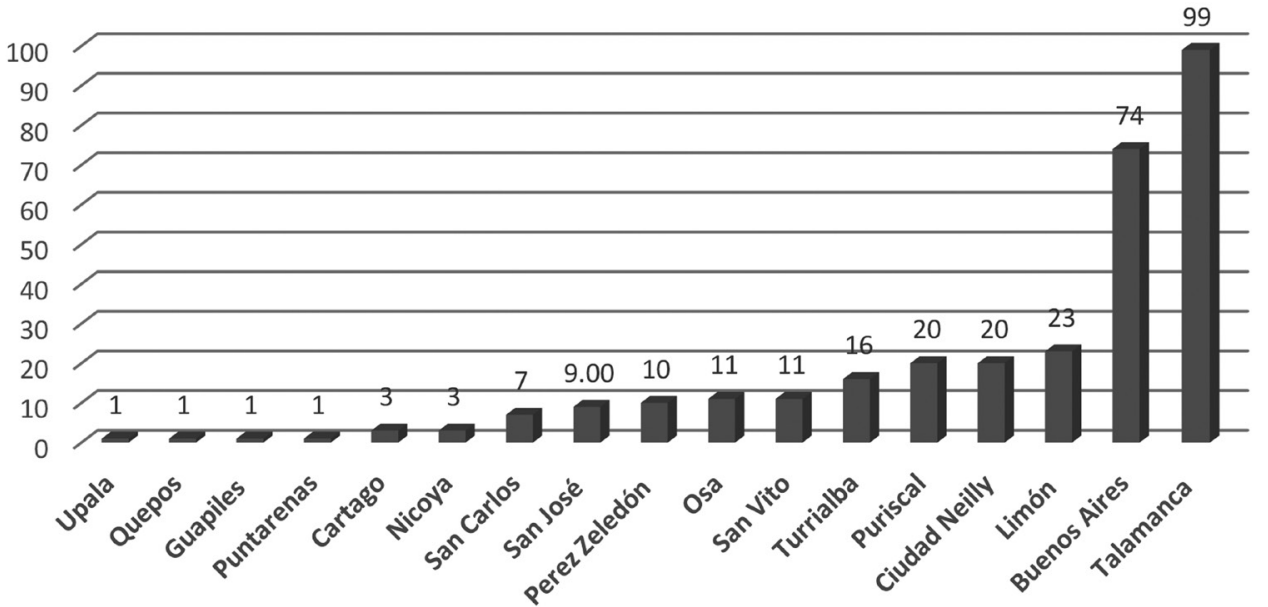

Figura 1. Estudiantes indígenas becados por condición socioeconómica, primer cuatrimestre 2017, según Centro Universitario. Fuente: Oficina de Atención Socioeconómica, mayo 2017. 


\section{Estudiantes privados de libertad}

La UNED ha realizado esfuerzos fundamentales para llevar educación a las personas privadas de libertad. Desde 1979 se crea un programa de atención a personas con esta condición y, en el 2004, se crea oficialmente el Centro Universitario en el Centro Penitenciario la Reforma, el más importante del país. En él se atienden estudiantes en 14 centros penales. Desde la Escuelas, la Dirección de Asuntos estudiantiles y Centros Universitarios se trabaja articuladamente en la atención de esta población, lo que se refleja en la tabla 11.

TABLA 11

Matrícula al primer cuatrimestre 2017 ordenado por centro penal

\begin{tabular}{|c|c|c|}
\hline Centro penitenciario & Cantidad hombres & Cantidad mujeres \\
\hline La Reforma & 50 & - \\
\hline Semi institucional & 29 & 15 \\
\hline Calle Real, Liberia & 33 & 1 \\
\hline San José, San Sebastián & 30 & - \\
\hline Pococí, Guápiles & 29 & - \\
\hline La Marina, San Carlos & 26 & - \\
\hline Virilla & 26 & - \\
\hline Sandoval, Limón & 20 & - \\
\hline Cocorí, Cartago & 18 & - \\
\hline San Rafael & 16 & - \\
\hline Pérez Zeledón & 14 & - \\
\hline Buen Pastor & - & 11 \\
\hline El Roble, Puntarenas & 4 & - \\
\hline Adulto Mayor & 4 & - \\
\hline Total & 299 & 27 \\
\hline
\end{tabular}

Fuente: elaboración propia con datos DAES, 2017.

TABLA 12

Estudiantes privados de libertad por género, primer cuatrimestre 2017

\begin{tabular}{lcc}
$\quad$ Género & Cantidad & Porcentaje \\
Masculino & 299 & 91 \\
Femenino & 27 & 9 \\
Total & 326 & 100 \\
\hline
\end{tabular}

Fuente elaboración propia con datos, DAES 2017. 


\section{Género en la UNED}

La UNED, en sus 40 años de quehacer académico, se ha convertido en una universidad fundamental para la mujer costarricense ya que, en su gran mayoría, los estudiantes de la UNED son mujeres. El contexto anterior se refleja en la tabla número 13, en donde se puede observar que, por medio de la educación a distancia, la mujer costarricense ha tenido grandes oportunidades de educación superior universitaria, así se refleja en la matrícula de los diferentes Centros Universitarios.

Como ejemplo, se concluye que, durante el año 2016, de los 32.239 estudiantes matriculados, el $63.18 \%$ de los estudiantes de la UNED son mujeres y el $36.82 \%$ hombres. En veintitrés centros universitarios, el porcentaje de mujeres sobrepasa el $65 \% \mathrm{y}$, de esos, diez centros sobrepasan el 70\% de estudiantes del género femenino.

Estos números dan a conocer la importancia que ha tenido la UNED con el acceso de las mujeres a la educación superior. Esto sin duda constituye un logro histórico que ha tenido alta incidencia en la sociedad costarricense y en la vida social, familiar y profesional de las mujeres en Costa Rica.

Viéndolo desde otra perspectiva, la mujer costarricense ha confiado en la UNED, para convertirse en profesional. Esta población ha encontrado, en la modalidad de educación a distancia, importantes oportunidades que ha valorado positivamente y que ya han sido reconocidas. La calidad académica que existe permite que los estudiantes de la UNED sean vistos como personas ordenadas, disciplinadas y responsables, ya que el modelo así lo exige; esas cualidades, llevadas al sector laboral, brindan grandes fortalezas a las personas como trabajadoras.

En el tema de género, es necesario resaltar la creación del Instituto de Estudios de Género de la UNED, establecido en Sesión del Consejo Universitario, 2005-2009 el 04 de noviembre del 2009, Artículo III, inciso 10. El instituto nació con la visión de orientar el quehacer de la UNED hacia el cambio a favor de la igualdad entre mujeres y hombres. Su misión se enfoca en la coordinación con las distintas dependencias de la institución y otras instituciones, tanto nacionales como internacionales, con el objetivo de desarrollar en la UNED, el eje de igualdad de género en forma transversal, en las distintas áreas sustantivas de la universidad.

TABLA 13

Estudiantes matriculados durante el 2016 durante el 2016 en la UNED por género

\begin{tabular}{lccccc}
\multicolumn{1}{c}{ Centro Universitario } & Femenino & $\%$ & Masculino & $\%$ & Total general \\
Acosta & 202 & 71,63 & 80 & 28,37 & 282 \\
Alajuela & 1323 & 61,39 & 832 & 38,61 & 2155 \\
Alajuela, sede interuniversitaria & 57 & 30,81 & 128 & 69,19 & 185 \\
Atenas & 104 & 64,60 & 57 & 35,40 & 161 \\
Atenas, sede interuniversitaria & 1 & 100,00 & & 0,00 & 1 \\
Buenos Aires & 286 & 70,79 & 118 & 29,21 & 404 \\
Cañas & 272 & 70,28 & 115 & 29,72 & 387 \\
\hline
\end{tabular}


Tabla 13 (Continuación)

\begin{tabular}{|c|c|c|c|c|c|}
\hline Centro Universitario & Femenino & $\%$ & Masculino & $\%$ & Total general \\
\hline Cartago & 1568 & 63,90 & 886 & 36,10 & 2454 \\
\hline Ciudad Neily & 315 & 63,89 & 178 & 36,11 & 493 \\
\hline Desamparados & 1043 & 63,79 & 592 & 36,21 & 1635 \\
\hline Estudiantes en el extranjero & 33 & 63,46 & 19 & 36,54 & 52 \\
\hline Privados de libertad & 21 & 10,05 & 188 & 89,95 & 209 \\
\hline Guápiles & 512 & 68,54 & 235 & 31,46 & 747 \\
\hline Heredia & 1627 & 61,96 & 999 & 38,04 & 2626 \\
\hline Jicaral & 91 & 75,83 & 29 & 24,17 & 120 \\
\hline La cruz & 112 & 65,50 & 59 & 34,50 & 171 \\
\hline La reforma & 2 & 2,13 & 92 & 97,87 & 94 \\
\hline Liberia & 389 & 65,05 & 209 & 34,95 & 598 \\
\hline Limón & 342 & 67,72 & 163 & 32,28 & 505 \\
\hline Monteverde & 79 & 79,80 & 20 & 20,20 & 99 \\
\hline Nicoya & 230 & 67,25 & 112 & 32,75 & 342 \\
\hline Orotina & 143 & 61,90 & 88 & 38,10 & 231 \\
\hline Osa & 157 & 78,11 & 44 & 21,89 & 201 \\
\hline Palmares & 1411 & 63,96 & 795 & 36,04 & 2206 \\
\hline Palmares, sede interuniversitaria & 9 & 90,00 & 1 & 10,00 & 10 \\
\hline Pavón & 92 & 63,45 & 53 & 36,55 & 145 \\
\hline Aula Puerto Jiménez & 55 & 88,71 & 7 & 11,29 & 62 \\
\hline Puntarenas & 435 & 65,41 & 230 & 34,59 & 665 \\
\hline Puriscal & 524 & 68,14 & 245 & 31,86 & 769 \\
\hline Quepos & 174 & 69,60 & 76 & 30,40 & 250 \\
\hline San Carlos & 893 & 66,94 & 441 & 33,06 & 1334 \\
\hline San Isidro de Pérez Zeledón & 994 & 69,12 & 444 & 30,88 & 1438 \\
\hline San José & 4642 & 57,45 & 3438 & 42,55 & 8080 \\
\hline San Marcos & 360 & 70,18 & 153 & 29,82 & 513 \\
\hline San Vito & 294 & 72,77 & 110 & 27,23 & 404 \\
\hline Santa Cruz & 189 & 63,64 & 108 & 36,36 & 297 \\
\hline Sarapiquí & 210 & 69,77 & 91 & 30,23 & 301 \\
\hline Siquirres & 226 & 70,63 & 94 & 29,38 & 320 \\
\hline Talamanca & 92 & 55,09 & 75 & 44,91 & 167 \\
\hline Tilarán & 108 & 76,60 & 33 & 23,40 & 141 \\
\hline Turrialba & 729 & 64,80 & 396 & 35,20 & 1125 \\
\hline Upala & 285 & 68,51 & 131 & 31,49 & 416 \\
\hline Total general & 20367 & 63,18 & 11872 & 36,82 & 32239 \\
\hline
\end{tabular}

Fuente: elaboración propia. 


\section{La UNED y la Educación Secundaria}

\section{Colegio Nacional de Educación a Distancia (CONED)}

Este proyecto de educación secundaria nació gracias a un convenio entre la UNED y el Ministerio de Educación Pública (MEP), firmado en julio del año 2005, cuando era Ministro de Educación el señor Manuel Solano Salas y el Rector de la UNED, Rodrigo Arias Camacho. El proyecto nació para costarricenses mayores de 18 años que, por diferentes razones de exclusión educativa y de falta de oportunidades, no habían logrado realizar sus estudios en el Tercer Ciclo de la Educación General Básica y en la Educación Diversificada.

Dado el éxito de la educación a distancia en la educación superior universitaria, se buscaba un proyecto con plena visión de inclusión educativa con un modelo similar, pero para la educación secundaria, en donde se les entregarían los materiales a los estudiantes para estudiar en sus casas, con tutorías presenciales, dos exámenes y dos tareas por asignatura, los estudios se realizan por semestres. Es así como el CONED fue creado por el Consejo Superior de Educación de Costa Rica, mediante el acuerdo número 02-27-04, adoptado en la sesión número 27-2004. Inicia sus labores en el 2005.

En este importante proyecto, el Ministerio de Educación Pública pagaría a los profesores y la UNED pondría a disposición del CONED las instalaciones de los centros universitarios. También, aportaría los gastos administrativos y los recursos humamos para atender al Colegio.

Lamentablemente, el 12 de diciembre del año 2012, siendo ministro de Educación Leonardo Garnier Rímolo, bajo la presidencia de Laura Chinchilla Miranda, se da un cierre técnico por parte del Ministerio de Educación Pública, apoyado por el Consejo Superior de Educación. El alegato era que los objetivos del CONED no se habían logrado, justificación que la UNED no compartía.

En este mismo sentido, era claro que la UNED no podría continuar con el proyecto, pues no contaba con los recursos económicos para la contratación de los profesores que corrían por cuenta del Ministerio de Educación Pública. Se habló de cierre técnico, por cuanto, lo que no se permitió más, fue la matrícula para nuevos estudiantes y el cierre total se daría hasta que los estudiantes actuales terminaran sus estudios.

Dichosamente, estos proyectos, que solo beneficios brindan al país, se resisten a morir. Por ello, el 27 de junio de año 2016, en la Administración del señor Luis Guillermo Solís Rivera, siendo Ministra de Educación la señora Sonia Marta Mora Escalante y Rector de la UNED don Luis Guillermo Carpio Malavasi, quien jugó un papel preponderante, es que se firma un nuevo convenio entre la UNED y el MEP para la reapertura del CONED. Con esto, se entiende el permitir de nuevo la matrícula para nuevos estudiantes, porque a la fecha de la firma de este nuevo convenio, el CONED aun contaba con más de 2000 estudiantes y, a la fecha del cierre técnico, tenía casi 5000 estudiantes.

La gran misión del CONED es llegar a personas de muy bajos recursos, ya que el monto que se cobra por asignatura es muy bajo, aproximadamente 10 dólares con todos los materiales didácticos. Posterior a sus estudios en el Colegio, la UNED se convierte en 
la Universidad que puede seguir brindado los estudios a estos estudiantes, que ya comprenden muy bien el modelo de educación a distancia.

En la actualidad, el CONED está en doce centros universitarios de la UNED y se proyecta la apertura de más sedes para seguir cumpliendo sus objetivos en todo el país. En la tabla 14 se muestran las sedes del CONED.

TABLA 14

Centros universitarios de la UNED que cuentan con el CONED

\begin{tabular}{rllc} 
& \multicolumn{1}{c}{ Sede } & Fecha/período de creación & Cantidad de estudiantes Primer semestre 2017 \\
1 & San josé & Febrero 2006 & 579 \\
2 & Cartago & Febrero 2006 & 173 \\
3 & Heredia & Febrero 2006 & 355 \\
4 & Palmares & Febrero 2006 & 246 \\
5 & Liberia & Febrero 2006 & 103 \\
6 & Nicoya & Febrero 2006 & 76 \\
7 & Turrialba & Febrero 2006 & 108 \\
8 & Limón & Febrero 2006 & 134 \\
9 & Ciudad neily & Febrero 2006 & 83 \\
10 & Alunasa & 24 de julio de 2006 & 132 \\
11 & Puntarenas & Febrero 2007 & 24 \\
12 & Acosta & Julio 2009 & 39 \\
& Total & & 2052 \\
\hline
\end{tabular}

Fuente: elaboración propia.

\section{Los colegios científicos de la UNED}

A inicios del 2000, bajo la rectoría del señor Rodrigo Arias Camacho (1999-2009), se da prioridad para la creación de instituciones orientadas a la formación de jóvenes en las áreas de la ciencia y la tecnología, con fundamento en la Ley de Promoción del Desarrollo Científico y Tecnológico9 (Ley 7169). En el 2002 y en el 2006, se concreta la creación de dos colegios de educación secundaria, que han venido a fortalecer y a liderar la educación media costarricense, con énfasis en la ciencia y tecnología en nuestro país: el Colegio Científico de Limón (CCL) y el Colegio Científico de Alajuela (CCAL). Esta labor fue asignada al señor Kenneth Rivera Rivera que, desde su fundación, ha fungido como Director Ejecutivo, llevando a cabo una excelente labor, graduando hasta el 2016, 448 bachilleres en educación media.

9. Ley 7169/1990, sobre Promoción del Desarrollo Científico y Tecnológico. (La Gaceta núm. 144 del 1 de agosto de 1990). 
TABLA 15

Total graduados al 2016

\begin{tabular}{cccc} 
Colegio & Cantidad de graduados & Hombres & Mujeres \\
CCL & 224 & $113(50.4 \%)$ & $111(49.6 \%)$ \\
CCAL & 224 & $134(59.8 \%)$ & $90(40.2 \%)$ \\
TOTAL & 448 & 247 & 201 \\
\hline
\end{tabular}

Fuente: elaboración propia con datos Colegios Científicos.

El objetivo de los Colegios Científicos (artículo 57, Ley 7169) «es la formación integral de sus estudiantes, considerando los más altos valores costarricenses en el marco de un proceso educativo, con énfasis en la adquisición de conocimientos sólidos y habilidades en los fundamentos de la matemática, la física, la química, la biología y la informática».

Los resultados obtenidos de los Colegios Científicos de la UNED en el último quinquenio (2012-2016) se reflejan en tres grandes ámbitos: a) las Pruebas Nacionales de Bachillerato; b) los puntajes de admisión a las universidades públicas y c) participación en eventos de ciencia y tecnología.

Con referencia a las Pruebas Nacionales de Bachillerato en Costa Rica, se muestran en la tabla 16, los resultados promedio para el quinquenio 2012-2016, en donde se aprecia que el Colegio Científico de Alajuela es el mejor promedio de Costa Rica entre más de 814 instituciones, con 95,46 en las pruebas escritas y con una nota de bachillerato de un 100,00. Por su parte, el Colegio Científico de Limón, ocupa el tercer mejor promedio nacional, con 93,33 y una nota de 99,46 respectivamente.

La promoción de estudiantes para ambos Colegios Científicos es del 100\% desde su creación. Se pueden observar los resultados obtenidos por las dos instituciones, comparándolos con los promedios del Sistema de Colegios Científicos de Costa Rica y los promedios nacionales para el mismo periodo. Se concluye que los dos Colegios Científicos de la UNED son líderes de la educación media costarricense. Paralelamente a estos resultados, varios estudiantes, tanto de Limón como de Alajuela, han obtenido los primeros promedios nacionales en bachillerato a nivel nacional.

TABLA 16

Indicadores Académicos (periodo 2012-2016)

\begin{tabular}{lcccccc} 
Institución & $\begin{array}{c}\text { Ranquin } \\
\text { CR }\end{array}$ & $\begin{array}{c}\text { Realizan Examen } \\
\text { (6 pruebas de bachillerato) }\end{array}$ & $\begin{array}{c}\text { Ganan } \\
\text { Examen }\end{array}$ & $\begin{array}{c}\text { Promedio } \\
\text { de examen }\end{array}$ & $\begin{array}{c}\text { Nota de } \\
\text { Bachillerato }\end{array}$ & $\begin{array}{c}\% \\
\text { promoción }\end{array}$ \\
CCAL & 1 & 24 & 24 & 95,09 & 100,00 & 100 \\
CCL & 3 & 14 & 14 & 93,33 & 99,46 & 100 \\
SNCCCR $\left(^{*}\right)$ & 7 & 17 & 17 & 91,97 & 98,81 & 99,87 \\
Costa Rica & $814(* *)$ & 44 & 26 & 68,52 & 80,16 & 61,00 \\
\hline
\end{tabular}

\footnotetext{
${ }^{(*)}$ Promedios del Sistema Nacional de Colegios Científicos.

${ }^{(* *)}$ Promedio de colegios de secundaria de Costa Rica para el periodo en estudio.

Fuente: elaboración propia con datos Colegios Científicos.
} 
En cuanto a los resultados sobre los puntajes de admisión a la Universidad de Costa Rica (UCR) y al Tecnológico de Costa Rica (TEC), los estudiantes de los dos Colegios Científicos de la UNED sobresalen con puntajes altos; el 100\% obtienen el derecho de ingreso. Vale agregar que el puntaje máximo en estas universidades es de 800 puntos.

En la tabla 17 se muestran los resultados promedio para el quinquenio 2012-2016. El promedio alcanzado por los estudiantes les permite no solo ingresar a las carreras de su preferencia, sino recibir becas, de acuerdo con su condición socioeconómica. El Colegio Científico de Alajuela es uno de los líderes nacionales en puntaje de admisión, tanto a la UCR como al TEC. En el 2016, un estudiante de Alajuela fue el que obtuvo 800 puntos a nivel nacional de la UCR. El promedio del quinquenio del Científico de Limón es de 650.9 puntos y 648.6 puntos, para la UCR y el TEC respectivamente. Para el Científico de Alajuela, es de 701.6 y 698.1 , respectivamente.

TABLA 17

Promedios de los Puntajes de Admisión en colegios científicos

\begin{tabular}{lcccc} 
Año & Colegio & Estudiantes & UCR & TEC \\
2012 & Limón & 14 & 627,3 & 647,5 \\
2013 & Limón & 12 & 680,4 & 653,5 \\
2014 & Limón & 10 & 613,0 & 598,7 \\
2015 & Limón & 15 & 654,4 & 656,7 \\
2016 & Limón & 19 & 679,2 & 686,4 \\
& & & & \\
2012 & Alajuela & 25 & 722,9 & 715,6 \\
2013 & Alajuela & 20 & 663,1 & 644,5 \\
2014 & Alajuela & 26 & 685,5 & 676,4 \\
2015 & Alajuela & 25 & 711,9 & 728,9 \\
2016 & Alajuela & 27 & 724,5 & 725,1 \\
\hline
\end{tabular}

Fuente: elaboración propia con datos de Colegios Científicos.

El tercer resultado académico, de impacto nacional, de los Colegios Científicos de la UNED lo constituyen los resultados obtenidos en la participación de eventos nacionales e internacionales, como las olimpiadas costarricenses de ciencias, matemática, de robótica, ferias de ciencias y tecnología, programas de obtención de pasantías y becas fuera del país, así como los bachilleres con excelencia que se obtienen cada año. En la tabla 18 se muestran los premios obtenidos en el quinquenio a nivel nacional e internacional. Los resultados del Colegio Científico de Alajuela en esta área son dominantes. En la actualidad, ambos colegios son el campeón y sub campeón nacional del Programa Antorcha (programa de televisión educativo), premio obtenido en el 2015. 
TABLA 18

Colegios Científicos de la UNED: Premios Obtenidos (2012-2016)

\begin{tabular}{lccccc}
\multicolumn{1}{c}{ Premio } & 2012 & 2013 & 2014 & 2015 & 2016 \\
Medallas ORO & 10 & 5 & 6 & 6 & 9 \\
Medallas PLATA & 5 & 5 & 7 & 4 & 13 \\
Medallas BRONCE & 14 & 10 & 12 & 12 & 12 \\
Menciones HONOR & 8 & 12 & 15 & 7 & 12 \\
Bachiller Excelencia & 14 & 26 & 30 & 28 & 34 \\
\hline
\end{tabular}

Fuente: elaboración propia con datos de Colegios Científicos.

\section{Cobertura de la UNED}

Otra visión con gran proyección de inclusión social fue la cobertura que la UNED se propuso, nació para estudiantes en todo el país, no solo para los de la gran área metropolitana. La cobertura también se había convertido en un factor de exclusión educativa, porque las universidades públicas estaban básicamente centralizadas en la gran área metropolitana. La idea de la UNED, entonces, se visualizaba con la apertura de sedes universitarias en todo el país.

El papel de las sedes universitarias ha sido fundamental para lograr los objetivos por los que fue creada la UNED. En el trascurso de estos 40 años, la evolución integral de los Centros Universitarios ha sido significativa y han sido ejes estratégicos para fortalecer el liderazgo de la universidad en las regiones, consolidando la expansión y la cobertura de la educación a distancia en el país.

Los centros universitarios, o mejor dicho, la UNED en las regiones, deben seguir fortaleciéndose para contribuir con el desarrollo integral de las diferentes comunidades. La vinculación con la comunidad es fundamental para seguir promoviendo importantes proyectos de extensión y acción social, así como de investigación en las diferentes regiones de Costa Rica, con principal atención en los cantones con bajos índices económicos y de desarrollo humano.

Al cumplir sus 40 años, la UNED cuenta con treintaiséis sedes universitarias en todo el país, factor fundamental, básico para la inclusión educativa. Además, cuenta con un aula descentralizada en la Palma de Puerto Jiménez, en la Zona sur, que depende del centro Universitario de Ciudad Neily. No se puede dejar de lado el Centro de Investigación Transferencia Tecnológica y Educación para el Desarrollo (CITTED), el cual fomenta proyectos de investigación y transferencia tecnológica y está ubicado en San Carlos, provincia de Alajuela. 
TABLA 19

Centros universitarios de la UNED, ordenados por año de creación

\begin{tabular}{|c|c|c|}
\hline & Centro Universitario & Año de Apertura \\
\hline 1 & San José & 01/07/1977 \\
\hline 2 & Puntarenas & 01/07/1977 \\
\hline 3 & Ciudad Neilly & 01/07/1977 \\
\hline 4 & La Cruz & $01 / 07 / 1977$ \\
\hline 5 & Osa & $10 / 11 / 1977$ \\
\hline 6 & Quepos & $01 / 07 / 1978$ \\
\hline 7 & Cartago & $01 / 07 / 1978$ \\
\hline 8 & Alajuela & $01 / 07 / 1978$ \\
\hline 9 & San Carlos & 01/07/1978 \\
\hline 10 & Palmares & 01/07/1978 \\
\hline 11 & Nicoya & $01 / 07 / 1978$ \\
\hline 12 & San Isidro & $01 / 07 / 1978$ \\
\hline 13 & Siquirres & 08/06/1978 \\
\hline 14 & Cañas & 08/07/1918 \\
\hline 15 & Limón & $01 / 11 / 1978$ \\
\hline 16 & Puriscal & 01/07/1979 \\
\hline 17 & Orotina & 01/08/1979 \\
\hline 18 & San Vito & $01 / 08 / 1979$ \\
\hline 19 & $\begin{array}{l}\text { La Reforma Como Programa De Atención } \\
\text { La Reforma Como Centro Universitario }\end{array}$ & $\begin{array}{l}1979 \\
2004\end{array}$ \\
\hline 20 & Guápiles & $10 / 10 / 1979$ \\
\hline 21 & Jicaral & $22 / 02 / 1980$ \\
\hline 22 & Upala & $01 / 05 / 1981$ \\
\hline 23 & Turrialba & $15 / 06 / 1981$ \\
\hline 24 & Liberia & 01/08/1981 \\
\hline 25 & San Marcos & $01 / 08 / 1982$ \\
\hline 26 & Santa Cruz & $01 / 02 / 1986$ \\
\hline 27 & Heredia & $20 / 05 / 1989$ \\
\hline 28 & Atenas & $17 / 06 / 1995$ \\
\hline 29 & Monteverde & 01/08/1998 \\
\hline 30 & Tilarán & $01 / 04 / 2002$ \\
\hline 31 & Sarapiquí & $15 / 01 / 2004$ \\
\hline 32 & Desamparados & $01 / 05 / 2004$ \\
\hline 33 & Pavón & $01 / 04 / 2005$ \\
\hline 34 & Talamanca & $28 / 04 / 2006$ \\
\hline
\end{tabular}


Tabla 19 (Continuación)

\begin{tabular}{|c|c|c|}
\hline & Centro Universitario & Año de Apertura \\
\hline 35 & Buenos Aires & $28 / 02 / 2011$ \\
\hline 36 & Acosta & 2010 \\
\hline 37 & Aula Desconcentrada De Ciudad Neily - Puerto Jiménez & 2004 \\
\hline 38 & Citted En San Carlos & 2007 \\
\hline
\end{tabular}

Fuente: elaboración propia con datos del CIDREB, centros universitarios y acuerdos del Consejo Universitario y Consejo de Rectoría.

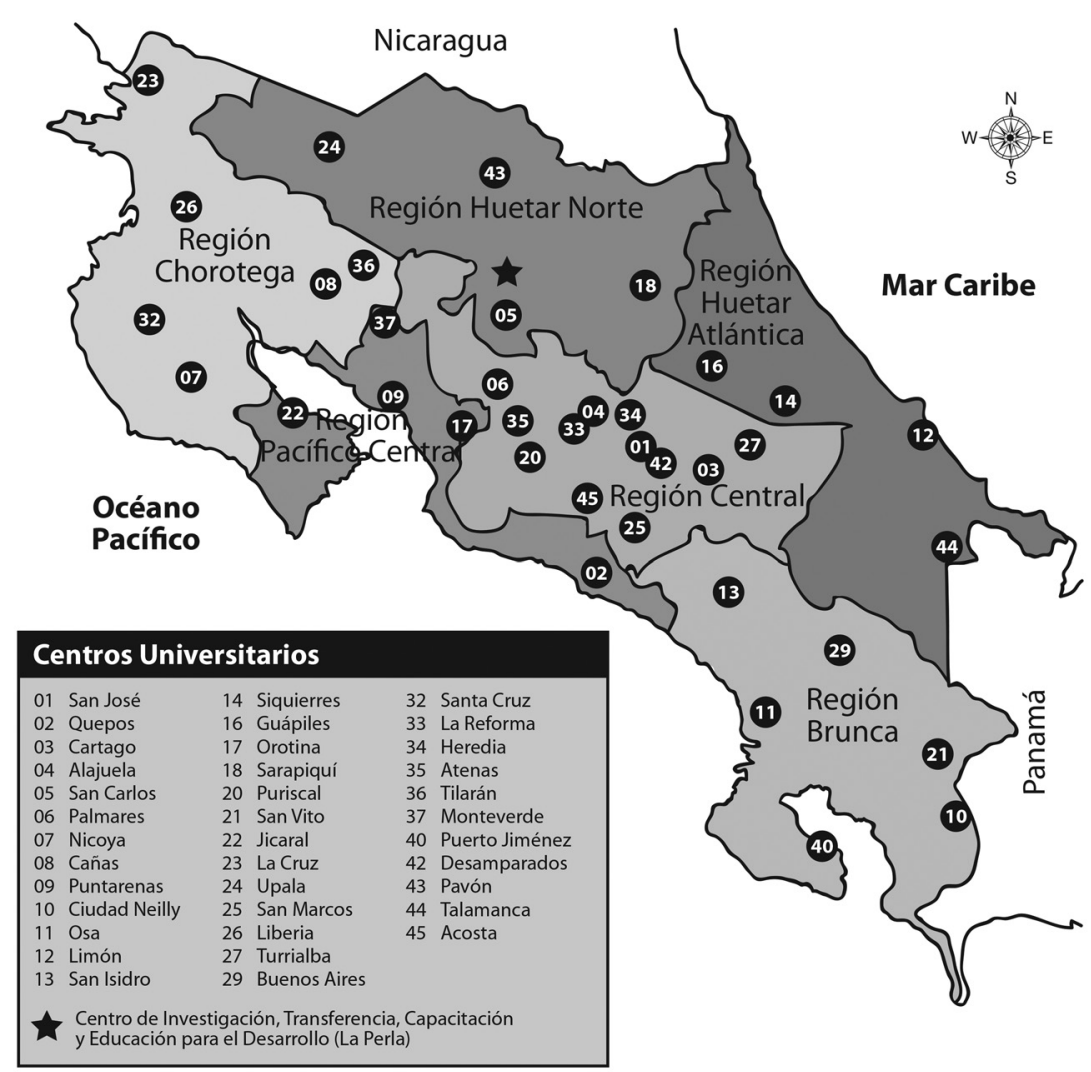

Figura 2. Mapa de cobertura de la UNED al 2017. Fuente: Centro de Planificación y Programación Institucional (2017). 
De esta manera, por el acceso y cobertura, en sus 40 años la UNED ha podido graduar a 54246 estudiantes, lo cual se refleja en la tabla número 20. Es importante señalar que, de los 54246 graduados, el $74 \%$ son mujeres y el 26\% hombres.

TABLA 20

Total de graduados a tercera promoción 2016

\begin{tabular}{lc} 
Graduados grado & 49075 \\
Posgrado & 2699 \\
Técnicos & 2472 \\
Total graduados & 54246 \\
\hline
\end{tabular}

Fuente: elaboración propia con datos de Oficina de Registro y CIEI al 2016.

\section{Calidad académica}

Gran parte del prestigio, a nivel nacional e internacional, tiene que ver con la apuesta en la calidad académica que la UNED ha emprendido con gran éxito. El trabajo conjunto entre la Vicerrectoría Académica, la Escuelas y el Instituto de Gestión de la calidad Académica (IGESCA), ha sido fundamental para llevar a cabo los procesos de autoevaluación y acreditación de las carreras por medio del Sistema Nacional de Acreditación (SINAES). Los procesos de autoevaluación son muy importantes para tener credibilidad en la sociedad.

Para que la calidad sea reconocida por la sociedad costarricense, en necesario acreditar las carreras. Por eso, la creación del Programa de Autoevaluación Académica (PAA), en el 2002 en la sesión 1560-2002 del Consejo Universitario, fue fundamental para el logro de los objetivos. Este Programa fue convertido en el Instituto de Gestión de la Calidad Académica en el 2015 en el acuerdo tomado por el Consejo Universitario, en sesión 2452-2015, Art. III, inciso 1-a celebrada el 13 de agosto del 2015.

En ese acuerdo, se le dan al Instituto como funciones: «investigar, orientar, planear, ejecutar, coordinar y difundir la cultura de la gestión de la calidad en la educación superior, a nivel institucional y coordinar con redes nacionales e internacionales».

Como Objetivo General se le dio:

Desarrollar investigación orientada a la indagación sistemática y rigurosa para el fomento de una cultura para el mejoramiento continuo en la oferta académica de la universidad, mediante la elaboración de lineamientos, procedimientos y el acompañamiento correspondiente que brinda en los procesos de autoevaluación, acreditación y mejoramiento de las carreras de grado, programas de posgrado y programas de extensión, y el desarrollo de proyectos específicos en materia de gestión de la calidad académica universitaria. 
TABLA 21

Carreras acreditadas en la UNED ante SINAES por Escuela en Pregrado, Grado y Posgrado a abril 2017

\section{Escuela de Ciencias de la Administración}

Diplomado en Administración de Empresas

Bachillerato y Licenciatura en Administración de Empresas con énfasis en Contaduría (reacreditada)

Bachillerato y Licenciatura en Administración de Empresas con énfasis en Banca y Finanzas

Bachillerato y Licenciatura en Administración de Empresas con énfasis en Recursos Humanos

Bachillerato y Licenciatura en Administración de Empresas con énfasis en Producción

Bachillerato y Licenciatura en Administración de Empresas con énfasis en Mercadeo

Bachillerato y Licenciatura en Administración de Empresas con énfasis en Dirección de Empresas

Escuela de Ciencias de la Educación

Bachillerato y Licenciatura en Informática Educativa (reacreditada)

Diplomado, Bachillerato y Licenciatura en Educación Preescolar

Bachillerato y Licenciatura en Administración Educativa

Bachillerato en Educación Especial

Diplomado, Bachillerato y Licenciatura en Educación General Básica, I y II Ciclos

Escuela de Ciencias Exactas y Naturales

Profesorado y Bachillerato en Enseñanza de la Matemática (por segunda vez reacreditada)

Profesorado y Bachillerato en Enseñanza de las Ciencias Naturales (reacreditada)

Bachillerato y Licenciatura en Manejo de Recursos Naturales (reacreditada)

Diplomado en Informática

Bachillerato y Licenciatura en Ingeniería Informática,

Licenciatura en Ingeniería Informática y Administración de Proyectos, Licenciatura en Ingeniería

Informática y Desarrollo de Aplicaciones Web, Licenciatura en Ingeniería Informática

y Calidad de Software.

Maestría en Psicopedagogía

Escuela de Ciencias Sociales y Humanidades

Diplomado, Bachillerato y Licenciatura en Enseñanza del Inglés para I y II Ciclos

Diplomado y Bachillerato en Gestión Turística Sostenible

En proceso de Acreditación ante SINAES

Bachillerato y Licenciatura en Administración de Empresa con énfasis en Negocios Internacionales

En Autoevaluación ante SINAES

Bachillerato y Licenciatura en Ingeniería Agronómica

Bachillerato y Licenciatura en Ingeniería Agroindustrial

Diplomado, Bachillerato y Licenciatura en Administración de Servicios de Salud

Licenciatura en Docencia

Licenciatura en Estudios Sociales y Educación Cívica

Maestría en Administración de Negocios

Maestría en Valuación

Maestría en Tecnología Educativa

Acreditado ante Agencia Centroamericana de Acreditación de Posgrado (ACAP)

Doctorado en Ciencias Naturales para el Desarrollo (Programa Interuniversitario)

Fuente: UNED, abril 2017. 
Cuando se habla de calidad académica, se hace necesario hablar del docente. Por eso, debe haber mucho énfasis en la calidad en la docencia. El papel del profesor en el mundo ya ha cambiado, ya no es suficiente solo impartir lecciones en un aula; los profesores deben apoyar en los procesos académicos de las universidades, generar investigación, escribir, publicar, dirigir tesis, utilizar plataformas virtuales, bases de datos, tutorías en línea, videoconferencias, entre otras actividades. Es la única manera de adaptarse a los cambios tecnológicos y colaborar con las personas que más requieren oportunidades reales para una inserción en la sociedad.

Por esta razón, ha sido muy importante la Creación del Centro de Capacitación en Educación a Distancia (CECED) para instruir a profesores, y profesionales en general, para que su labor sea más eficiente. El CECED se creó en el 2000, en sesión del Consejo de Rectoría 1142-2000. Se fundó como parte de la Vicerrectoría Académica y su misión está enfocada en ofrecer capacitación y formación a los profesionales de la universidad, especialmente a los que contribuyen en los procesos docentes ofrecidos por la UNED.

\section{Calidad en la gestión Universitaria}

En muchas universidades, cuando se habla de calidad, solo se alude a la de tipo académico y se deja de lado la calidad en la gestión universitaria como apoyo a la académica. Es así como desde la Vicerrectoría de Planificación de la UNED se ha realizado una propuesta para la creación de un Programa de la Calidad de la Gestión Universitaria.

Tal como conceptualiza la Comisión Nacional de Evaluación y Acreditación Universitaria $^{10}$, la gestión institucional está compuesta por un conjunto de factores en los que se incluyen los procesos y recursos. Estos, necesariamente, deben estar al servicio y contribuir positivamente al desarrollo de las áreas sustantivas de la universidad: la docencia, la investigación y la extensión.

La UNED, desde hace varios años, como ya se indicó, inició los procesos de mejoramiento en sus programas académicos para los procesos de autoevaluación y acreditación. Muchas de estas acciones corresponden a la mejora en los procesos de gestión y administrativos y que, en el marco de las orientaciones académicas, trata de apoyar para alcanzar los objetivos propuestos por la academia.

La gestión universitaria como apoyo a la academia debe verse desde una perspectiva sistémica. Los procesos académicos deben comprenderse como todas aquellas acciones, estrategias y mecanismos interrelacionados entre sí, que deben ser apoyados por una gestión universitaria, vinculada, interconectada y complementaria. Toda la experiencia en estos procesos de calidad ha permitido determinar que la gestión de una Institución de Educación Superior como la UNED, constituye un factor clave en los procesos de mejoramiento y calidad universitaria, particularmente en la ejecución de los objetivos educativos de cada una, apoyando y facilitando el desarrollo ellos.

10. CONEAU. Evaluación de la Gestión Universitaria. Argentina: Comisión Nacional de Evaluación y Acreditación Universitaria. 2000. 
El contexto anterior conlleva a plantear la importancia de crear un programa de la calidad de la gestión universitaria que permita consolidar y coordinar, estrategias y acciones una gestión universitaria de calidad, en una gestión que cumpla tanto con la misión de la universidad y el contexto nacional, como con los más altos estándares internacionales, tomando en cuenta experiencias de otras universidades y las normas internacionales en materia de calidad en la gestión universitaria. Esto significa plantear la calidad en correspondencia con los propósitos declarados de la institución y consolidar un conjunto de criterios de calidad que establezca estándares ideales a los cuales debe aspirar la UNED.

De esta manera, la institución debe plantear como objetivo general:

Liderar a nivel institucional la consolidación y sostenibilidad de un Sistema de Calidad en la Gestión universitaria que involucre a todas las áreas de la universidad. Es imprescindible consolidar una gestión institucional de excelencia sustentada en los resultados, calidad y la mejora continua de las dependencias universitarias. También, propiciar un modelo de gestión de la calidad para la universidad, que incluya los lineamientos, prácticas y criterios de calidad para la gestión de la universidad, fortaleciendo las capacidades y mecanismos de gestión de la universidad sobre sus distintos procesos.

Dentro de los principales objetivos que debería tener el Programa de Calidad en la Gestión universitaria, se pueden mencionar los siguientes:

1. Propiciar un modelo de gestión de la calidad para la universidad, que incluya lineamientos, prácticas y criterios.

2. Coordinar y liderar los procesos de autoevaluación, mejoramiento y certificación de calidad de los procesos institucionales, promoviendo la mejora de la calidad institucional, fortaleciendo las capacidades y mecanismos de gestión de la universidad sobre sus distintos procesos.

3. Establecer un modelo de evaluación de la gestión universitaria que, en materia de calidad, incluya evaluar los servicios universitarios.

4. Diseñar el manual de Calidad Institucional basado en los objetivos institucionales y los requerimientos internacionales y nacionales sobre la gestión universitaria.

5. Consolidar indicadores estratégicos de calidad, centrados en la efectividad, pertinencia e impacto de la universidad.

6. Articular, coordinar y vincular las acciones de mejora institucional desde la vicerrectoría de Planificación con los diferentes actores institucionales en el marco del fortalecimiento de la gestión universitaria.

7. Coordinar con el IGESCA las acciones de mejoramiento derivadas de los procesos de acreditación de las carreras que competen a nivel de la gestión institucional.

8. Diseñar y ejecutar estrategias de socialización, capacitación y divulgación de una cultura de calidad en todas las instancias de la UNED. 
9. Coordinar con las instancias responsables aquellas prácticas y acciones innovadoras orientadas hacia la calidad y el mejoramiento continuo de la gestión universitaria.

10. Brindar el seguimiento y monitoreo necesario a las políticas de calidad, los objetivos y planes de mejoramiento institucional, para lograr su cumplimiento.

11. Propiciar, con las instancias responsables, la conformación de equipos de gestión de la calidad, que den seguimiento a las acciones de calidad.

\section{El teletrabajo como factor de innovación de la gestión universitaria y de inclusión social.}

El teletrabajo se ha convertido es una modalidad que cumple importantes objetivos en las sociedades, promoviendo la inclusión social. Además, mejora la calidad de vida de las personas trabajadoras, aumenta la productividad y disminuye los costos fijos en las organizaciones. En un mundo tan complejo como el actual y con tanto congestionamiento vial, es definitivo que el teletrabajo disminuye los niveles de estrés y brinda grandes aportes al mejoramiento de la movilidad en las ciudades, además reduce los índices de contaminación.

La Universidad Estatal a Distancia ha visto el teletrabajo como una nueva forma de innovar en la gestión institucional por lo que ha establecido importantes proyectos en esta temática, con la finalidad de lograr altos niveles de desempeño en los servicios que brinda a la sociedad costarricense.

En este mismo sentido, la UNED ha sido la primera universidad pública de Costa Rica que se acogió el decreto ejecutivo No. 34.704 «Promoción del Teletrabajo en las Instituciones Públicas» del 11 de febrero del 2013. Ya antes había realizado un plan piloto en el 2008, con 16 trabajadores.

Oficialmente, es el 1 Consejo de Rectoría de la UNED, en acuerdo tomado en sesión No. 1729-2012, Artículo V, inciso 3, celebrada el 25 de junio de 2012, el que establece el Programa de Teletrabajo como un proyecto a desarrollar desde la Vicerrectoría de Planificación y, a su vez, acuerda conformar la Comisión Institucional de Teletrabajo en el acuerdo de la sesión No. 1733-2012, Artículo VI, celebrada el 23 de julio de 2012.

Esta Comisión, con representación de diferentes dependencias de la institución, se dio a la tarea de conceptualizar y diseñar la Carta Constitutiva del Teletrabajo en la UNED y el Reglamento del Teletrabajo de la UNED. Así, el Consejo Universitario, en enero del 2015 (sesión 2397-2015, Art. II, inciso 1-a, celebrada el 22 de enero del 2015 y aprobado en firme en la sesión 2402-2015), aprueba el Reglamento de Teletrabajo y constituye formalmente el Programa de Teletrabajo, adscrito a la Vicerrectoría de Planificación.

Dada la creación del programa, bajo la coordinación de la señora Adriana Oviedo Vega, y en conjunto con la Comisión, se inicia un intenso trabajo. En abril del 2015, se abre a la comunidad universitaria la convocatoria para que los funcionarios que deseaban incorporarse a esta modalidad laboral, iniciaran el proceso. 
Como una decisión estratégica, la Comisión decidió capacitar, previamente a esa convocatoria, a todas las jefaturas institucionales, incluyendo así tanto a coordinadores, jefes, directores, vicerrectores y hasta el rector. Esa capacitación se realizó de manera bimodal, lo que implicó una sesión presencial y luego un curso virtual por medio de la plataforma Moodle. Se abordaron aspectos laborales, legales, tecnológicos, médicos y de salud ocupacional. Actualmente, la UNED cuenta con 132 funcionarios, con personal a cargo, capacitados en materia de teletrabajo. Igualmente, las personas teletrabajadoras han tenido que ser capacitadas para lograr éxito en sus funciones y en la modalidad en general. En la tabla 22, se da a conocer información importante sobre el teletrabajo en la UNED.

TABLA 22

Información general del trabajo en la información general del trabajo en la UNED a mayo 2017

Total personas teletrabajadoras en la UNED

152

Teletrabajadores en el extranjero

Porcentaje de mujeres teletrabajando

Porcentaje de hombres

Promedio de edad

42

Cantidad de días promedio de días de teletrabajo

2

Principales días de teletrabajo

Lunes

Puestos que tienen más personas teletrabajadoras

Productores académicos, Profesores universitarios Analistas de sistemas.

Fuente: elaboración propia con datos del Programa de Teletrabajo (2017).

Para valorar realmente el impacto de la modalidad de teletrabajo en la UNED, la Vicerrectoría de Planificación llevó a cabo una investigación que fue respondida por 72 personas teletrabajadoras. Dicha pesquisa arrojó importantes conclusiones que se detallan en la tabla número 23 .

TABLA 23

Criterios ofrecidos por los teletrabajadores de la UNED

\begin{tabular}{lc}
\multicolumn{1}{c}{ Variables } & $\%$ \\
Indicó como muy alto el aumento de su productividad. & $57 \%$ \\
Indicó como alto el aumento de su productividad. & $35.4 \%$ \\
Manifestó que tuvo una muy alta mejora en la atención y calidad & $62 \%$ \\
de los servicios que brinda. & $93.7 \%$ \\
Calificó con muy alto el nivel de concentración. & $73.4 \%$ \\
Indicó con muy alto el uso de las TIC para el desarrollo de sus funciones.
\end{tabular}




\begin{tabular}{lc}
\multicolumn{1}{c}{ Variables } & $\%$ \\
$\begin{array}{lc}\text { Valoró con muy alto el efecto en la planificación y organización del tiempo y en el } \\
\text { seguimiento y control de su trabajo. }\end{array}$ & $70.9 \%$ \\
Calificó como muy alto las mejoras en el ambiente laboral. & $58.2 \%$ \\
Manifestó con muy alto el ahorro económico por concepto de traslado y combustible. & $78.5 \%$ \\
Valoró con muy alto las mejoras en su calidad de vida y el balance trabajo-familia. & El $87.3 \%$ \\
Indicó con muy alto las mejoras en las prácticas en alimentación y ejercicios. & $62 \%$ \\
\hline
\end{tabular}

Fuente: elaboración propia con datos de Investigación Vicerrectoría de Planificación 2016 por Rosberly Rojas.

Es una realidad, entonces, que el teletrabajo ha propiciado ahorros al teletrabajador; ahorro a la institución en energía eléctrica, agua; ha colaborado también con el uso del parqueo institucional; además de proporcionar un efecto positivo en el ambiente, disminuyendo la producción de CO2. Es importante agregar que el Programa de Teletrabajo adscrito a la Vicerrectoría de Planificación ha establecido otros objetivos de valor agregado que pretenden posicionar a la UNED como líder en esta modalidad y se visualiza el Teletrabajo como un proyecto país, por lo que está desarrollando proyectos en tres líneas: teletrabajo para privados de libertad, telecentros y capacitaciones; y asesorías en el tema de teletrabajo al sector empresarial y público en todo el país.

\section{Conclusiones}

En sus 40 años, la Universidad Estatal a Distancia ha demostrado ser inclusiva; este objetivo fue establecido desde su misión en 1977, año en que fue creada. La educación a distancia ha democratizado la educación superior en el mundo y la UNED ha jugado un papel preponderante en la educación costarricense.

Ha quedado demostrado que la UNED ha apostado por el acceso, cobertura y calidad como tres grandes pilares de inclusión educativa. Con respecto al acceso, ha sido fundamental no tener examen de admisión, brindando, de esta manera, oportunidades de estudio a las personas que han sido por mucho tiempo excluidas de las universidades presenciales. A su vez, la UNED también se ha convertido ya en la primera alternativa que muchos estudiantes buscan para cursar sus estudios.

El acceso lo ha demostrado, también, brindando servicios a poblaciones específicas como personas con alguna discapacidad, poblaciones indígenas, privados de libertad y la educación secundaria con el proyecto del Colegio Nacional de Educación a Distancia y los colegios científicos. Aunado a ello, con respecto al género, en su gran mayoría sus estudiantes son mujeres que, por medio de la UNED, han podido tener grandes oportunidades que antes de 1977 no tenían.

Aun cuando la UNED es la universidad que menos recursos recibe del FEES, se ha convertido en la segunda universidad en población estudiantil, atendiendo un promedio 
anual de 32.000 estudiantes. Esta institución ha realizado enormes esfuerzos por contar con un programa fortalecido de becas que han bridado un gran apoyo a las personas de muy bajos recursos.

Con respecto a la cobertura, la UNED ha quebrado los esquemas que han mantenido otras universidades públicas del país, ya que esta no solo ha ubicado sus sedes mayoristamente en la gran área metropolitana. Se ha preocupado, más bien, por abrir sedes universitarias en todos los rincones de Costa Rica. Actualmente, sus 36 centros universitarios, posicionan a la universidad en muchas regiones. La UNED está ubicada desde el Océano Pacifico hasta el Mar Caribe, y desde la frontera con Panamá hasta la Frontera con Nicaragua. Eso sí, se deben seguir haciendo esfuerzos mayores en las regiones porque mayoritariamente la población estudiantil está ubicada en la gran área metropolitana. Además, en el fortalecimiento de las regiones se debe tener como objetivo que las personas no migren a la capital y permanezcan en sus comunidades brindando importantes aportes a las regiones.

En el tema de calidad académica, la Universidad ha logrado alta credibilidad, al apostar por la autoevaluación y acreditación de sus carreras. Este proceso es sumamente costoso; sin embargo, la UNED ha invertido grandes recursos que, junto al gran trabajo de la academia, ha logrado acreditar casi el $60 \%$ de sus programas. Gracias a todas estas evidencias, se puede contar con la certeza de que la educación a distancia por medio de la UNED se ha convertido en un factor de inclusión social, una institución de educación inclusiva.

En el tema de gestión universitaria, se han presentado proyectos de calidad para la mejora de la gestión como apoyo a la academia y, a su vez, se ha convertido en la primera universidad pública que ha implementado la modalidad de teletrabajo. El Programa de Teletrabajo no solo ha pensado en enviar funcionarios a teletrabajar, a nivel país tiene importantes proyectos ya que se ha propuesto ser una institución líder en este tema que se considera fundamental para Costa Rica, brindando de esta manera soluciones laborales.

En otro artículo, se abordarán los temas de pertinencia de la oferta, permanencia del estudiante, uso de las TIC en la educación y la equidad en servicios. Todos ellos factores fundamentales, necesarios para poder hablar de educación inclusiva. Igualmente, con el tema de la internacionalización, necesaria para el trabajo conjunto con otras universidades del mundo. Cuarenta años han pasado y se han logrado importantes objetivos, pero la UNED debe seguir buscando la mejora continua en todos sus procesos académicos y de gestión para seguir brindando servicios de calidad a la razón de ser de nuestra institución: el estudiante.

La educación a distancia es equidad y oportunidades, la educación a distancia es inclusión social, la educación a distancia ha democratizado la educación en el mundo. 


\section{ABSTRACT \\ Distance Education as Social Inclusive Factor: UNED, 40 Years Democratizing Education in Costa Rica}

Distance education has become a teaching method which provides great opportunities to students all over the world who; for many years, have not had access to higher conventional education. In Costa Rica, there was no knowledge about this form of education until 1977 when a university with a distance education method opened its doors. In this article, the reader will find a summary of the work the Distance State University of Costa Rica has carried out during 40 years with a vision of social inclusion.

Key words: social inclusion, distance education, coverage, equity, quality.

\section{RÉSUMÉ}

\section{L'enseignement à distance comme facteur d'inclusion sociale: UNED, 40 ans en démocratisant l'enseignement au Costa Rica}

L'enseignement à distance est devenu une forme d'enseignement qui offre des grandes opportunités aux étudiants du monde qui n'ont pas eu d'accès à l'éducation supérieur en présentiel. Au Costa Rica cet forme d'étudier est connue jusqu'à l'année 1977 avec l'ouverture d'une université d'enseignement à distance. Cet article fait connaître, en bref, le travail qui a réalisé l'Universidad Estatal a Distancia (UNED) - Université de l'État à Distance - du point de vue de l'inclusion sociale.

Mots-clés: Inclusion sociales, enseignement à distance, couverture, équité., qualité.

\section{Bibliografía}

Booth, Tony, «Manteniendo el futuro con vida; convirtiendo los valores de la inclusión en acciones. Rompiendo inercias. Claves para avanzar». VI Jornadas Científicas de Investigación sobre Personas con Discapacidad (2006): 211-217).

Booth Tony y Mel Ainscow. «Index for inclusión». Developing leaning and participation in schools. $3^{\mathrm{a} e d .}$ Manchester: CSIE, 2011.

Carpio-Malavasi, Luis Guillermo. Informe de Labores UNED 2016. Costa Rica: Universidad Estatal a Distancia, 2017.

Casanova, María Antonieta y Miguel Ángel Cabra de Luna. Educación y personas con discapacidad. Presente y Futuro. Madrid, España: Fundación ONCE, 2009.

CONEAU. Evaluación de la Gestión Universitaria. Argentina: Comisión Nacional de Evaluación y Acreditación Universitaria. 2000.

Echeita, Gerardo, «Inclusión y exclusión Educativa: Voz y Quebranto». Revista Iberoamericana sobre Calidad, Eficacia y Cambio en Educación, n² (2013):9-18.

Comisión Europea. «Informe conjunto sobre la inclusión social en el que se resumen los resultados del examen de los planes nacionales de acción en favor de la inclusión social (2003-2005)». Bruselas, Bélgica: Comisión Europea, 2003.

Silver Hilary y S. M. Miller, «Social Exclusion. The European Approach to Social Disadvantage». Indicators, $\mathrm{n}^{\circ} 2$ (2003): 7-11.

Ley 6044/1977 de Creación de la Universidad Estatal Distancia (La Gaceta núm.50 del 12 de marzo de 1977).

Ley 7169/1990 sobre Promoción del Desarrollo Científico y Tecnológico. (La Gaceta núm. 144 del 1 de agosto de 1990). 